\title{
La agricultura familiar campesina en Centroamérica: una apuesta estratégica frente a los desafíos de los territorios rurales ${ }^{\prime}$
}

Ileana Gómez, Rafael Cartagena, Xenia Ortiz y Oscar Díaz Investigadores de la Fundación Prisma

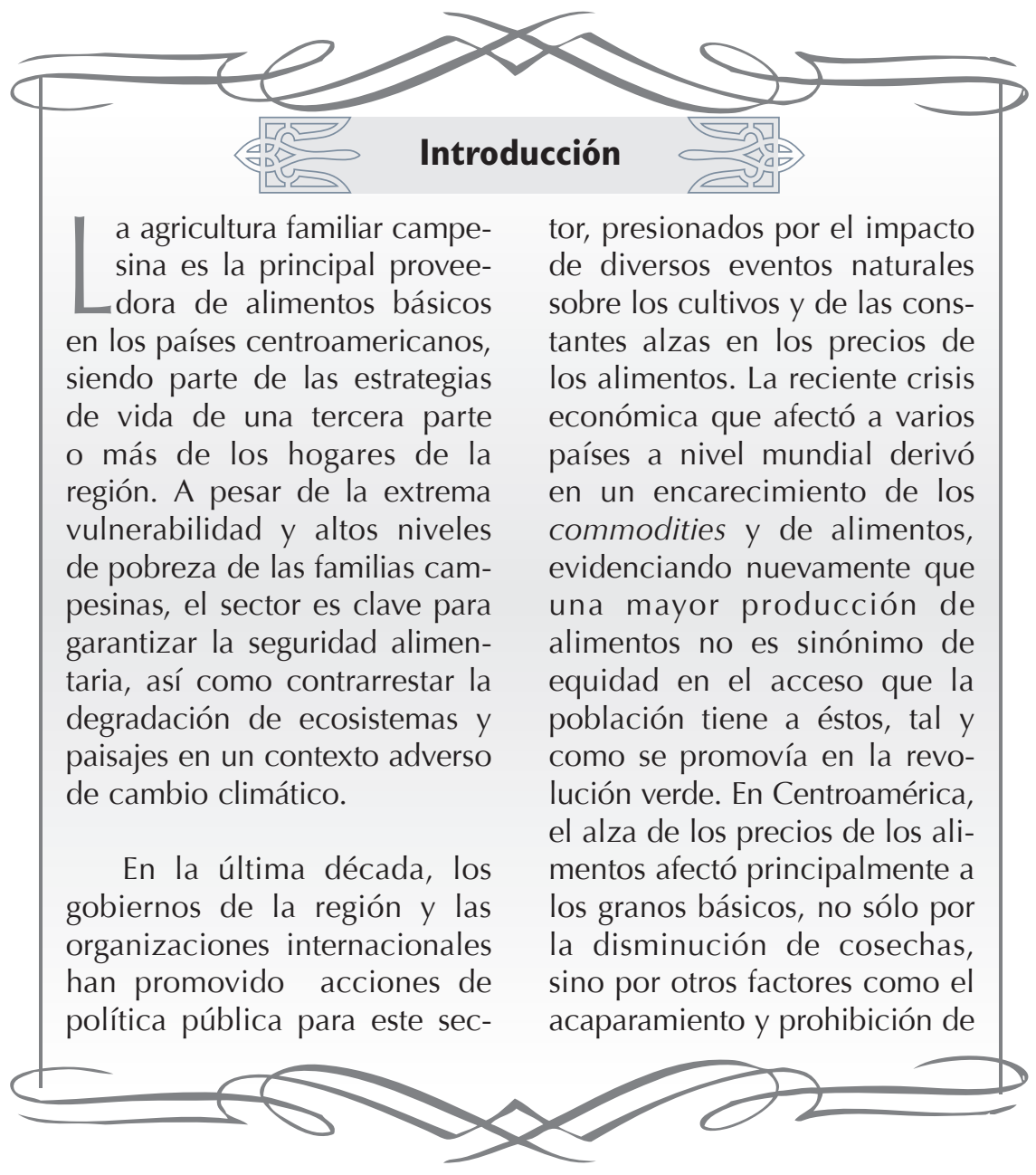


exportaciones. Esta alza de precios repercute en los pequeños productores de granos básicos que ya son vulnerables debido a sus condiciones de pobreza (van der Zee Arias, 2012).

Los esfuerzos desde la política pública se dirigen a procurar la alimentación de las familias rurales, buscando incrementar la producción nacional de alimentos para garantizar la seguridad alimentaria y nutricional así como la estabilidad económica en momentos de crisis (Peraci Sanchez, 2011).

Las limitaciones y el contexto adverso para la agricultura familiar campesina se profundizan por el cambio económico. En las últimas décadas, las políticas de liberalización comercial han afectado la producción familiar en dos sentidos: en primer lugar, el mercado de granos básicos de la región se ha vuelto más complejo, castigando a los pequeños; además, la liberalización ha favorecido un auge exportador de algunos productos agroindustriales produciendo conflictos que presentan amenazas para los derechos de las poblaciones campesinas e indígenas, como el desplazamiento y desalojo de población y los efectos de la contaminación derivada de la producción agroindustrial.

Aun con esta situación poco favorable, una ventaja de la región -todavía poco aprovechada- es que cuenta con marcos estratégicos regionales para agricultura, desarrollo rural territorial, recursos naturales y cambio climático, y con mecanismos de participación que permiten la interacción de diversos actores como organizaciones de productores, centros de generación de conocimiento, gobiernos locales y agencias de desarrollo, para promover políticas más integrales y efectivas que procuren un nuevo rol para el sector en el marco del desarrollo de los territorios rurales.

\section{Importancia de la agricultura familiar campesina en la economía bienestar: Dimensiones de la agricultura familiar campesina}

Además de proveer la mayoría de los alimentos de la dieta básica de la región, la agricultura familiar campesina tiene un peso demográfico y económico considerable, sumando más de 2,1 millones de unidades productivas desde Guatemala hasta Nicaragua, equivalente a una tercera parte de los hogares de esta región (conocida como CA4), en la que representaría por lo menos el $64 \%$ de los ocupados en el sector agropecuario y el $49 \%$ del PIB agropecuario.

En los países del CA-4 se registran alrededor de 3,2 millones de personas con ocupación principal en el sector agropecuario, tomando en cuenta asalariados, patronos, trabajadores por cuenta propia y familiares no remunerados. ${ }^{2}$ Las dos últimas 
categorías coinciden, a grandes rasgos, con los criterios que definen la agricultura familiar, y representan algo más de 2 millones de personas desde Guatemala a Nicaragua. En términos porcentuales, ambas categorías representan un $62 \%$ de los ocupados del sector en Guatemala, un $50 \%$ en El Salvador, $76 \%$ en Honduras y $64 \%$ en Nicaragua.

Algunos segmentos de la agricultura familiar campesina no están visibilizados en esta categorización. Es el caso de pequeños productores que los registros oficiales contabilizan como "patronos". Otro segmento no considerado está formado por productores cuya ocupación principal se realiza en ramas ajenas a la actividad agropecuaria, sin embargo cultivan una parcela a nivel familiar, generalmente granos básicos. Se estima que en la región CA-4 comprenden más de 770 mil productores (PESA/FAO, 2011).
La agricultura familiar campesina es parte de las estrategias de vida de una tercera parte o más de los hogares de la región. En Guatemala esto alcanza un $48 \%$ de los hogares. Honduras y Nicaragua presentan valores muy similares, de $30 \%$ y $34 \%$ respectivamente, mientras que la cifra más baja se aprecia en El Salvador $(13,4 \%){ }^{3}$

La información registrada por las encuestas de hogares permite estimar los ingresos generados por la agricultura familiar campesina, y con ello hacer un cálculo de su aporte al PIB agropecuario. ${ }^{4}$ Los ingresos de las fincas encabezadas por un trabajador por cuenta propia y los ingresos del estrato de micro-empleadores representarían el $41 \%$ y $8 \%$, respectivamente, del valor agregado agropecuario en los 4 países. El otro $51 \%$ correspondería al valor agregado por medianos y grandes empleadores (empresarios, empresas agrícolas, cooperativas) (Baumeister, 2011).

\section{Gráfico 1}

\section{Aporte de la agricultura familiar al PIB agropecuario. En porcentajes}

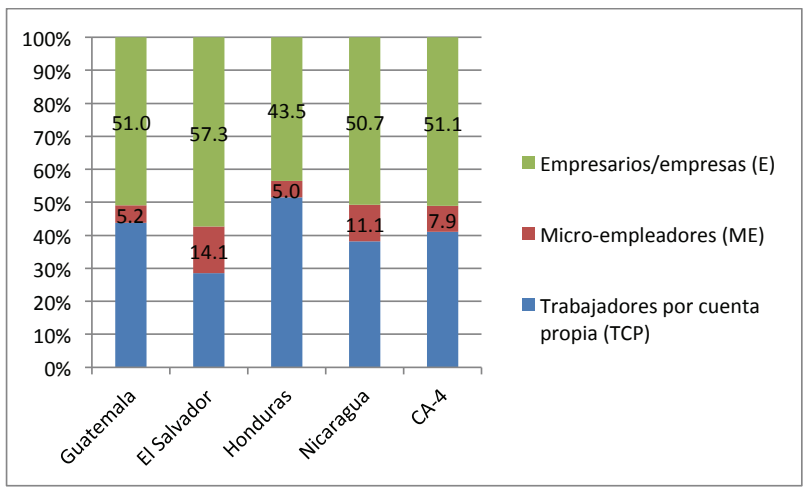

Fuente: (Baumeister, 2011) 
La agricultura familiar campesina en Centroamérica se caracterizaría por encontrarse casi en su totalidad en el estrato de subsistencia. De acuerdo a (Eitzinger, y otros, 2012), "para el segmento de productores que dependen de la agricultura para sus medios de vida, la degradación de los recursos naturales ${ }^{5}$ y la baja producción de maíz/frijol están íntimamente relacionadas con los determinantes principales de la pobreza, entre ellos: el aislamiento geográfico, la falta de acceso a servicios e infraestructura, al crédito y los mercados de insumos y productos; los bajos niveles de educación y la dependencia de la mano de obra familia".

La agricultura familiar de subsistencia (AFS) ${ }^{6}$ alcanza el $76 \%$ de las explotaciones en Nicaragua pero solamente el $21 \%$ de la tierra agropecuaria, mientras que en El Salvador la AFS alcanzaría el y $86 \%$ de las explotaciones y un $17 \%$ de la tierra.
Una característica propia de la AFS de la región de Centroamérica es el reducido tamaño de las explotaciones, con una media de 1 ha en Nicaragua y 0.7 ha en El Salvador. En El Salvador se ha notado una "polarización extrema" de la distribución de la tierra, pues las fincas mayores de 100 ha manejan un $41,3 \%$ de toda la tierra agropecuaria (Maletta, 2012).

Esto implica que una política pública que privilegie a los segmentos en "transición" y "consolidada" estaría excluyendo a la gran mayoría de productores familiares, y con ellos una tercera parte de los hogares de la región en mayor condición de vulnerabilidad. Más aún, estas políticas deben tomar en cuenta la limitada dotación y mala calidad de la tierra a la que tiene acceso la agricultura familiar en su conjunto, en especial en el segmento de subsistencia.

\section{Agricultura familiar campesina en tiempos de crisis y cambio}

La agricultura familiar campesina en Centroamérica, tal como ha sido caracterizada en el apartado anterior, se desarrolla en un contexto de inseguridad alimentaria agudizada por los impactos de la variabilidad climática. Las limitaciones y el actual contexto adverso para la agricultura familiar campesina se profundizan por el cambio económico relacionado con la apertura de las economías centroamericanas a la globalización.? Procesos de distribu- ción y reconcentración han ocurrido en la estructura agraria debido a las reformas agrarias y programas de transferencias de tierra. Estas reformas significaron que unas 290.000 familias campesinas (el $23 \%$ de las familias rurales) accedieran a la tierra (Baumeister, 1999). Sin embargo, los programas de reforma agraria se detuvieron para volver al predominio de los mecanismos de mercado (Merlet, 2004), abriendo la puerta a intereses de empresas nacionales 
y transnacionales. Una de las consecuencias de esta debacle fue la rápida venta y fragmentación de la propiedad del sector reformado.

En un contexto marcado por políticas de liberalización comercial, las tierras reformadas se convierten en un preciado recurso para nuevas inversiones. De acuerdo a Cuellar (2012), el crecimiento reciente de nuevos flujos de inversión es el resultado de la "fiebre por la tierra" derivada de procesos de aumento en la demanda mundial de alimentos y materias primas, y del surgimiento de un mercado creado en función de la demanda de tierra para la producción de biocombustibles.

Entre 1990 y 2007, la inversión extranjera directa en el sector agrícola se triplica y emergen nuevas modalidades de inversión como la agricultura por contrato, el arrendamiento de tierras y la compra adelantada de cosechas (Cuellar, 2012). La expansión de cultivos para la agroindustria de exportación está configurando nuevas y más complejas formas de relación en la tenencia de la tierra. Una parte de estos nuevos agentes toma tierras en arriendo no sólo de grandes terratenientes sino de pequeños productores y campesinos, muchos de ellos beneficiarios de la reforma agraria que no están en condiciones de costear los insumos necesarios para mantener una producción rentable en estos rubros. En Gua- temala se han detectado casos de arrendamiento de esa naturaleza para hortalizas de exportación, estableciendo relaciones de arriendo y proporcionando empleo asalariado a los pequeños propietarios de tierra (CEPAL, 2001). En El Salvador, las plantaciones de caña de azúcar en el Bajo Lempa se han extendido sobre tierras alquiladas por empresas cañeras a pequeños productores -que lo ven como una forma más fácil y menos riesgosa de obtener ingresos- y a cooperativas que dejaron de ser sembradas colectivamente y se parcelaron entre sus socios. Buena parte de la tierra que solía ser alquilada para granos básicos, pero también la destinada a introducir otras alternativas productivas como la agro-forestería, ya se está destinando al cultivo de caña de azúcar (PRISMA, 2012).

\subsection{Incidencia de las políticas de liberalización comercial en la agricultura familiar campesina}

Las políticas de liberalización comercial han afectado la producción familiar en dos sentidos: en primer lugar, el mercado de granos básicos de la región se ha vuelto más complejo, castigando con más dureza a quienes cuentan con menos información y conocimiento para negociar precios; en este caso, los pequeños productores agropecuarios. Por otra parte, la liberalización ha favorecido un auge exportador de algunos productos agroindustriales, lo cual estaría 
llevando a un desplazamiento de la producción familiar de granos básicos en determinados territorios de la región.

El proceso de liberalización comercial regional y la consiguiente correlación de precios de un país a otro han ensanchado las asimetrías de información entre los productores locales, los intermediarios que compran el producto en finca $y$ los exportadores/importadores. En un contexto de incertidumbre, los distintos actores de la cadena de comercialización buscan protegerse del riesgo, siendo el pequeño productor el que se encuentra en mayor desventaja al momento de negociar precios con el comprador en finca, ${ }^{8}$ debido a su escaso o nulo conocimiento de las tendencias del mercado regional.

\subsection{Agricultura familiar campesina, nuevas inversiones $y$ disputas en los territorios}

La política comercial también está incentivando la siembra de cultivos en función del mercado exterior, lo cual presenta amenazas para la agricultura familiar, ya sea por un incremento en las dificultades en acceder a la tierra vía compra o renta, o por un proceso de pérdida de los derechos de usufructo y posesión de comunidades campesinas e indígenas.

En la mayoría de países centroamericanos se verifica la expan- sión de cultivos de caña de azúcar o palma africana. El acuerdo comercial con los EE.UU. ha permitido incrementar las exportaciones de azúcar, dulces, confites y similares ha dicho país.

Pero diversos conflictos han surgido a raíz del avance de estas inversiones por la amenaza a los derechos de las poblaciones campesinas e indígenas, principalmente relacionadas con el desplazamiento y desalojo de población y con los efectos de la contaminación derivada de la producción agroindustrial. De acuerdo a Cuellar (2011), "crecientemente los territorios indígenas y las tierras en manos de comunidades campesinas, están bajo la mirada de actores económicos nacionales, regionales y transnacionales para el desarrollo de proyectos diversos en zonas rurales. Los procesos de regularización, así como los mercados de tierra han pasado a ser instrumentos claves para posibilitar el acceso de inversionistas a importantes extensiones de tierra para la implementación de inversiones en zonas que antes eran dedicadas a la producción campesina".

Las organizaciones campesinas e indígenas se movilizan para demandar nuevas reglas de juego. Según las organizaciones campesinas hondureñas, la Ley para la Modernización y Desarrollo del Sector Agrícola (1992) ha permitido el acaparamiento de tierras, dando como resultado los actuales conflictos 
agrarios, el incremento de pobreza, la injusticia rural y la aguda conflictividad social del campo hondureño, que tiene su máxima expresión de violencia en el Bajo Aguán. En Guatemala, donde hay una histórica conflictividad agraria, existe una fuerte presión de las organizaciones campesinas e indígenas para que sea aprobada la Ley de Desarrollo Rural Integral, y con esta medida exigen que se permita el acceso a la tierra. Esta conflictividad ha agudizado la militarización de comunidades, desalojos de colonos y campesinos de sus tierras de cultivo, así como ejecuciones extrajudiciales de sus líderes (Zagema, 2011), para dar paso a las inversiones de palma africana o caña de azúcar.

En El Salvador, los movimientos sociales en el Bajo Lempa no están en contra de la producción de caña, pero demandan la prohibición de la quema y el riego aéreo de madurantes por los impactos negativos de la contaminación en la salud de la población y en la conservación de ecosistemas como los manglares, de suma importancia para la economía local y la gestión del riesgo en la zona costera.

Al hacer un análisis desde los territorios es más claro evidenciar cómo la agricultura familiar campesina convive con las nuevas inversiones ya sea en relaciones de conflicto o de articulación (ofreciendo fuerza de trabajo, participando en contratos de alquiler, produciendo insumos etc.), aunque esto no supone que se realice en condiciones ventajosas que permitan dinámicas para la mejora sectorial y territorial a mediano o largo plazo. Pero a diferencia de la crisis alimentaria y climática, estos conflictos no son temas integrados explícitamente en las políticas relacionadas a la agricultura familiar campesina, a pesar que estos han moldeado y seguirán moldeando las particularidades del sector, adquiriendo diversas características en los territorios de la región.

\subsection{Vulnerabilidad de la agricultura familiar campesina frente al cambio climático}

Otro elemento crítico es la variabilidad climática. De todas las regiones tropicales, Centroamérica es el punto más vulnerable al cambio climático, cuyos impactos amenazan con socavar las bases del desarrollo en una región altamente expuesta y vulnerable debido a su condición socio-económica (Gutierrez, 2010).

Los recursos clave para los medios de vida de las familias campesinas están siendo afectados por la variabilidad climática, expresada en la mayor propensión a eventos meteorológicos extremos, sequías e inundaciones, que afecta las zonas geográficas donde predominan las actividades vinculadas a la agricultura familiar campesina, como la producción de granos básicos, 
siembra de hortalizas y productos de traspatio. La franja pacífica de la región está más expuesta a la amenaza de la sequía, en tanto en el atlántico la amenaza principal son las inundaciones (Ver Mapa 1).

Centroamérica ya muestra los graves efectos de la confluencia de la crisis alimentaria y los efectos del cambio climático. En Guatemala, el gobierno decretó estado de calami- dad pública en septiembre de 2009 al enfrentar la severa crisis alimentaria provocada por la variabilidad climática expresada en una severa sequía y por los efectos de las tormentas tropicales de 2005 y 2009. ${ }^{9}$ El alza de precios de los alimentos y otras consecuencias de la crisis económica, como la caída de remesas, contribuyeron a agudizar la crisis alimentaria que afectó a 410.780 familias pobres. ${ }^{10}$

Mapa 1

Centroamérica: uso de suelo, inundaciones y sequías

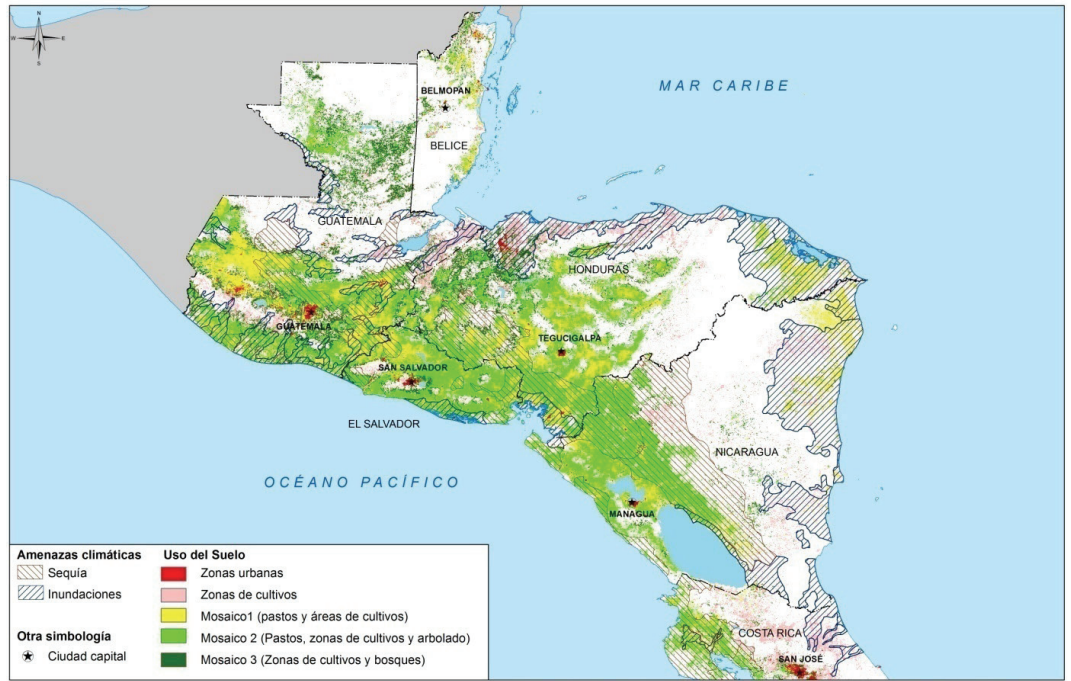

Fuente: Elaborado por PRISMA con base en OXFAM (mapa de amenazas, 1998) y CATHALAC (mapa de uso del suelo, 2007).

En El Salvador, en 2010, las pérdidas por inundaciones en la agricultura superaron los US\$100 millones, mientras las pérdidas por sequía sumaron US\$38 millones. Estos eventos afectaron la cosecha record de granos básicos que se esperaba como resultado de la implementación del Programa de Agricultura Familiar (PAF), con el cual el gobierno ha buscado reactivar la producción agrícola campesina. 
Otros efectos de la variabilidad climática en la agricultura familiar campesina es la incidencia negativa en los rendimientos de los principales granos básicos (Eitzinger, y otros, 2012) y la previsible presión sobre ecosistemas sensibles, tales como bosques y humedales ante la degradación de las zonas de cultivo.

\section{Enfoques y preocupaciones desde las políticas públicas}

Revalorar la agricultura familiar desde las políticas es clave para enfrentar los retos de la seguridad alimentaria, el cambio climático y la sostenibilidad ambiental. Ante esta situación crítica, los gobiernos e instituciones de cooperación han ido construyendo una serie de medidas de políticas y programas orientados a proteger la seguridad alimentaria y nutricional de la región. ${ }^{11}$ Instituciones como FAO y FIDA han señalado la gran contribución de la agricultura familiar a la seguridad alimentaria de la población (Schneider, 2009), (FAO, 2010), (Schejtman, 2008 ), y estas ideas han permeado en el desarrollo de sus programas en la región, especialmente el Programa Especial para la Seguridad Alimentaria (PESA) y diversos proyectos de FIDA.

En general, los programas nacionales mantienen un enfoque sectorial, se concentran en la producción de alimentos, brindando insumos, asistencia técnica -basada en los criterios de la revolución verde-, y promoción del acceso a mercados. Están enfocados en grupos que viven en condiciones de pobreza, con los que se prioriza alcanzar la seguridad alimentaria y, en la medida en que esto se logre, se puede avanzar a la generación de excedentes para salir de la pobreza.

Al nivel de las políticas regionales, Centroamericana cuenta con marcos que tienen un enfoque integrador de la seguridad alimentaria, la gestión sostenible de los recursos, la gobernanza y el desarrollo territorial. Varias estrategias regionales han captado la complejidad de los cambios económicos y ambientales de las últimas décadas. Las más notables, la Estrategia Centroamericana de Desarrollo Rural Territorial (ECADERT), con un fuerte proceso participativo, y la Estrategia Regional Agroambiental y de Salud (ERAS). Son acuerdos políticos al nivel del SICA, aprobados por todos los países de Centroamérica, que pretenden armonizar las políticas intersectoriales para que sean equitativas y ambientalmente sostenibles.

Otro elemento relevante es el rol que están jugando las políticas de cambio climático y gestión de riesgo. Los países han tenido que fortalecer sus acciones para garantizar la seguridad alimentaria más 
allá de los momentos de emergencia. Hay una corriente que se abre a vincular lo agrícola con lo ambiental y lo territorial, donde las estrategias de adaptación al cambio climático adquieren relevancia. Para el caso, el Proyecto Regional del Corredor Seco Centroamericano ${ }^{12}$ aborda el problema de la sustentabilidad de la producción campesina, asegurando la alimentación en el contexto de la amenaza climática de la sequía, y conlleva el impulso de prácticas agroecológicas y de la Ilamada agricultura inteligente. ${ }^{13}$

Recientes programas nacionales enfatizan la restauración de los ecosistemas y agro-ecosistemas, lo que implica impulsar nuevos sistemas de producción, estrategias para la innovación, gestión de conocimiento, fortalecimiento del tejido organizativo local y renovar los sistemas de extensión. En El Salvador, se está desarrollando el Programa de Restauración de Ecosistemas y Paisajes (PREP), liderada por el Ministerio de Medio Ambiente y Recursos Naturales. El PREP es un esfuerzo de adaptación al cambio climático con fuerte ancla en tres territorios de la principal cuenca del país, el rio Lempa; con un enfoque de paisaje, busca construir resiliencia frente a la variabilidad climática y facilitar las condiciones para conservar la diversidad biológica (MARN, PNUD, 2012).

\section{Conclusiones}

En Centroamérica, la agricultura familiar campesina vuelve a ser tomada en cuenta por la crisis del precio de los alimentos y por la creciente amenaza de la variabilidad climática, que se expresa en sequias y eventos meteorológicos extremos que ya están impactando negativamente la producción de los granos básicos indispensables para la dieta familiar.

Siendo la principal proveedora de granos básicos y dado su considerable peso demográfico y económico, asegurar la sostenibilidad de la agricultura familiar campesina se vuelve clave para la estabilidad social, económica y en general para la gobernanza a diversos niveles. $\mathrm{Si}$ bien los planes y programas nacionales se han enfocado en garantizar que haya una respuesta a una situación de crisis, masificando las medidas como los paquetes agropecuarios (pensados en respuesta a la emergencia de precios o a contrarrestar el efecto negativo de los eventos extremos), no se están definiendo estrategias que permitan la viabilidad socioeconómica de la agricultura familiar, sobre todo la de subsistencia.

El enfoque de las políticas para responder a la conjugación de estas crisis no basta para un sector que ha entrado con gran desventaja 
frente a la liberalización comercial en cuanto a información, acceso a créditos, infraestructura y conocimientos, y que al mismo tiempo vive en una relación de disputa y rearticulaciones con las nuevas inversiones agroindustriales. Esta compleja dinámica no está siendo considerada por las actuales políticas relacionadas a la agricultura familiar campesina, a pesar de que están moldeando las particularidades del sector en diversos territorios de la región.

\section{Bibliografía}

os Angel, A. (2008). Análisis de mercado de granos básicos en Centroamérica: enfoque en El Salvador. Programa Mundial de Alimentos. Disponible en http://www.amyangel.webs.com/ESfinal.pdf.

os Ayala, E. (1 de Diciembre de 2012). Cooperativas centroamericanas piden incidiren política ambiental. Recuperado el 25 de Enero de 2013, de IPS: http://ipsnoticias.net/nota.asp?idnews=102002

os Baumeister, E. (2010). Características económicas y sociales de los agricultores familiares en América Central. Documento Borrador.

os Baumeister, E. (2011). Características económicas y sociales de los agricultores familiares y aspectos de la evolución del comercio agropecuario y alimentario entre los países de América Central. FAO-RUTA.

os Baumeister, E. (1999). Las iniciativas campesinas y los resultados de la sostenibilidad de la reforma agraria en El Salvador, Honduras y Nicaragua. Discussion Paper no. 105.

os Baumeister, E. (2010). Pequeños productores de granos básicos en América Central. Honduras: FAO-RUTA.

os CEPAL (2001). La estructura agraria y el campesinado en El Salvador, Guatemala y Honduras. México.

os Consejo Agrícola Centroamericano (2008). Estrategia Regional Agroambiental y de Salud de Centroamérica. San José: CAC.

os Consejo Agrícola Centroamericano (2007). Política Agrícola Centroamericano 2008-2017: Una agricultura competitiva e integrada para un mundo global. San José: CAC.

os Consejo Agropecuario Centroamericano (2010). Estrategia Centroamericana de Desarrollo Rural Territorial (ECADERT). San José: CAC.

os Cuellar, N. (2012). Inversiones y dinámicas territoriales en Centroamérica. Implicaciones para la gobernanza y la construcción de alternativas. San Salvador: Fundación Prisma. 
os Cuellar, N. S. (2011). Dinámicas Territoriales en Centroamérica: Contexto y desafíos para comunidades rurales. Cuadernos PRISMA, 27.

os Eitzinger, A., Läderach, P., Sonder, K., Schmidt, A., Sain, G., Beebe, S., y otros (2012). Tortillas en el comal: Los sistemas de maíz y fríjol de América Central y el cambio climático. Cali, Colombia: CIAT (Centro Internacional de Agricultura Tropical).

os FAO (2010). Agricultura Climáticamente Inteligente: Políticas, prácticas y financiación para la seguridad alimentaria, adaptación y mitigación . Roma: FAO.

os FAO (2009). PESA FAO. Disponible http://www.pesacentroamerica.org/ Guatemala/noticias/especial_guatemala.php

os FAO, R. D. (enero de 2011). Caso de la experiencia del Bono Productivo Agropecuario: Hambre Cero. Disponible http://www.ruta.org/Documentos-CD/ExpereinciasSistematizadas/PDF/NICARAGUA_CasoBonoProductivoAgropecuario.pdf

os FAO-BID (2007). Políticas para la Agricultura Familiar en América Latina y el Caribe. Chile: Oficina Regional de la FAO para América Latina y el Caribe.

os FIAN Internacional (Coord.) (2010). El derecho a la alimentación en Guatemala. Informe Final Misión Internacional de Verificación. Ciudad de Guatemala: Magna Terra Editores.

os FIDA (2005). Información de evaluación ex ante. San Salvador: FIDA.

os Gobierno de Reconciliación y Unidad Nacional (2012). Plan Operativo Anual PRORURAL Incluyente. Disponible http://www.magfor.gob.ni/ prorural/IMesa2012/POASectorial2012.pdf

os Gutierrez, M. (2010). Vulnerabilidad y adaptación al cambio climático. Diagnóstico inicial, avances, vacios y potenciales líneas de acción en Centroamerica. Washington: BID. Notas Técnicas.

os Luna, F. (2012). Revisión de las políticas públicas sobre seguridad alimentaria y fomento de la producción en la agricultura familiar, en el marco de un proyecto de incidencia regional en Centroamérica (Guatemala, Honduras, Nicaragua, El Salvador). San Salvador: RIMISP-PRISMA.

os MAG (23 de Jan de 2013). MAG. Obtenido de http://www.mag.gob.sv

os MAG (2012). Plan Sectorial de Agricultura Familiar 2011-2014. San José, Costa Rica. 
os Maletta, H. (2011). Tendencias y perspectivas de la agricultura familiar en América Latina. Chile: RIMISP (Centro Latinoamericano para el Desarrollo Rural).

os Maletta, H. (2012). Tendencias y perspectivas de la agricultura familiar en América Latina. Argentina.

os MARN, PNUD (2012). El Programa Nacional de Restauración de Ecosistemas y Paisajes. Un esfuerzo principal de adaptación al cambio climático en El Salvador del Ministerio de Medio Ambiente y Recursos Naturales. Documento elaborado por Deborah Barry, MARN, San Salvador.

os Merlet, M. (2004). Fragilidad y límites de las reformas agrarias en America Central: las enseñanzas de dos países: Honduras y Nicaragua. BIVICAT- RECCAT, FAUSAC; URACCAN.

os Ministerio de Agricultura, Ganadería y Alimentación (14 de enero de 2013). Programa de Agricultura Familiar para el fortalecimiento de la economía campesina (PAFFEC) 2012 - 2016. Ciudad de Guatemala. Obtenido de http://www.dialogo.gob.gt/docs/programa_de_agricultura_familiar.pdf

os Oldeman, L. R. (1991). World map of the status of human-induced soil degradation: an explanatory note.Second revised edition. Global Assessment of Soil Degradation (GLASOD). Wageningen, Netherlands; International Soil Reference and nformation Centre (ISRIC), Wageningen, Netherlands; and United Nations Environmental Programme (UNEP).

os Peraci Sanchez, A. (2011). Agricultura Familiar: Evolución Conceptual, desafíos e institucionalidad. Lima: FAO, Iniciativa América Latina y Caribe sin Hambre.

os PESA/FAO (2011). Centroamérica en Cifras: Datos de Seguridad Alimentaria Nutricional y Agricultura Familiar. Programas Especiales para la Seguridad Alimentaria (PESA) de FAO Centroamérica.

os PRESANCA II-FAO (2011). Centroamérica en Cifras. Datos de seguridad alimentaria nutricional y agricultura familiar. IALCSH-PESA.

os PRISMA (2012). Análisis participativo de los detonantes de la degradación en el Bajo Lempa. Diagnóstico Jaltepeque. San Salvador: Fundación Prisma.

os Rosa, H. (2009). Perfiles y trayectorias del cambio económico en Centroamérica. Una mirada desde las fuentes generadoras de divisas. San Salvador: Fundación Prisma.

os Schejtman, A. (2008). Alcances sobre la agricultura familiar en América Latina. Chile: RIMISP (Centro Latinoamericano para el Desarrollo Rural). 
os Schejtman, A. (2008). Alcances sobre la agricultura familiar en América Latina. Documento de trabajo No.21, Programa Dinámicas Territoriales Rurales. Santiago, Chile: RIMISP.

os Schneider, S. (2009). A Pluriatividade na Agricultura Familiar. Porto Alegre. Brasil: Editora UFRGS.

os Secretaría de Agricultura y Ganadería de Honduras (s.f.). Estrategia del Sector Público Agroalimentario y Plan de Implementación. Disponible http://www.sag.gob.hn/assets/display-anything/gallery/1/65/Estrategia-delSector-Publico-Agroalimentario.pdf

os van der Zee Arias, A. v. (2012). Estudio de caracterización del Corredor Seco Centroamericano, Tomo I. Tegucigalpa: FAO.

os Zagema, B. (2011). Land and Power. The growing scandal surrounding the new wave of investments in land. Land and Power. The growing scandal surrounding the new wave of investments in land. Disponible en: http://www.oxfam.org/en/grow/policy/land-and-power.

\section{Notas}

1 Preparado en el marco del Proyecto de

6 (FAO-BID, 2007) propuso clasificar Incidencia Regional sobre Agricultura Familiar campesina. Centro Latinoamericano de Desarrollo Rural RIMISP.

2 Según cifras de los Censos de Población de Guatemala (2002), Honduras (2001) y Nicaragua (2005), citadas en Baumeister (2011). Para El Salvador, se usaron datos de la Encuesta de Hogares.

3 Como referencia, en Costa Rica, esta cifra no supera el $7 \%$.

4 El valor agregado agropecuario utilizado para dicha comparación corresponde a la reportada por el informe Estado de la Región del año 2008, según se indica en (Baumeister, 2011).

5 De acuerdo a (Oldeman, 1991), citado por (Eitzinger y otros, 2012) "se reportó en 1991 que el $75 \%$ de toda la tierra de vocación agrícola en los países del CA-4 está degradado. La tierra se ha degradado aun más desde entonces, pero no se ha llevado a cabo un estudio completo de estudios en 20 años". las explotaciones familiares en tres segmentos: Agricultura Familiar de Subsistencia (AFS), cuenta con una insuficiente dotación de recursos para el sustento familiar; Agricultura Familiar en Transición, (AFT), accede a tierras y recursos en mayor calidad y cantidad, pero tiene dificultades para generar excedentes; y Agricultura Familiar Consolidada (AFC), que se encuentra en mejores condiciones de acceso a tierra de calidad, créditos, tecnología, mercados y genera excedentes para la capitalización de la unidad productiva.

7 En 1978, la agro-exportación tradicional representaba entre el 74\% (Nicaragua) y el $65 \%$ (Honduras); para el 2006 había pasado a representar entre el $18 \%$ (Nicaragua) y el $4 \%$ en El Salvador, mientras se expanden otro tipo de exportaciones, sobre todo productos agrícolas no tradicionales, maquila, servicios y las remesas producidas por la emigración (Rosa, 2009). 
8 El precio que recibe un productor suele estar fijado por el comprador en finca, conocido en varios lugares de la región como "el coyote". Los excedentes de la mayoría de productores no justifican la inversión en transporte para llevar el producto a los mercados locales, ya no se diga metropolitanos.

9 Específicamente por los efectos de las tormentas tropicales Stan en 2005 y Aghata en 2009 la cual causó pérdidas en la producción agrícola en 16 departamentos; mientras la sequía provocada por el fenómeno El Niño destruyó el $90 \%$ de la producción de alimentos en el país (FIAN Internacional (Coord.), 2010).

10 Sólo en 2009 más de 54 mil de estas familias, ubicadas en el llamado corredor seco del este y noreste de Guatemala, fueron declaradas en estado crítico por la falta de alimentos debido a la pérdida de más de 36 mil has de cosechas de maíz y fríjoles, básicos para su dieta alimenticia (FAO, 2009).

11 Entre estos el Programa de Agricultura Familiar para el Fortalecimiento de la
Economía Campesina (PAFFEC) en Guatemala, el Plan de Agricultura Familiar (PAF) en El Salvador y Hambre Cero en Nicaragua.

12 El proyecto oficialmente se denomina "Aumento de la capacidad de resiliencia de los medios de vida de los pequeños productores/as frente a la sequía en el corredor seco centroamericano". Es producto de un convenio entre la Fundación Acción contra el Hambre (ACF) y la Organización de las Naciones Unidas para la Alimentación y la Agricultura (FAO), con apoyo financiero del Departamento de Ayuda Humanitaria y Protección Civil de la Unión Europea (ECHO, por siglas en inglés), (ACF, FAO, ECHO, SF).

13 Se ha establecido el Marco Estratégico Regional para la Gestión del Riesgo Climático en el Sector Agrícola del Corredor Seco (MERGERCA), orientado a resolver problemas agrícolas y de manejo de recursos naturales enfocado en los pequeños productores expuestos a la sequía. Su implementación está vinculada al Consejo Agropecuario Centroamericano (CAC). 